\title{
PROPIEDADES MECÁNICAS DE CONCRETOS MODIFICADOS CON PLASTICO MARINO RECICLADO EN REEMPLAZO DE LOS AGREGADOS FINOS
}

\author{
Jhon Fishman Venitez-Mosquera ${ }^{1,3}$, Yuliana Marcela Córdoba-Palacios ${ }^{1,3}$, Karol Patricia Mena- \\ Ramírez $^{1,3}$, Oscar Felipe Arbelaez Pérez ${ }^{2,3}$ \\ ${ }^{1}$ Semillero de investigación en materiales y energía. \\ ${ }^{2}$ Grupo de investigación termomec. \\ ${ }^{3}$ Universidad Cooperativa de Colombia.
}

Correo de correspondencia: oscar.arbelaez@campusucc.edu.co

\section{RESUMEN}

Los desechos plásticos marinos y su baja tasa de reutilización contribuyen a la contaminación ambiental, requiriéndose estrategias de valorización. Este trabajo presenta el diseño y la preparación de mezclas de concreto tradicional y con reemplazos del $2.5 \%, 5 \%, 7.5 \%$ y $10 \%$ de los agregados finos por plásticos reciclados del mar. A partir de las mezclas preparadas se elaboraron especímenes cilíndricos de $15 \mathrm{~cm} \times 30 \mathrm{~cm}$ de diámetroxaltura, respectivamente. Se evaluó el asentamiento, la densidad, la resistencia y el modulo elástico. Los resultados mostraron que un aumento del contenido de plástico reciclado genera una disminución del asentamiento y la densidad, así como un aumento de la resistencia y del módulo elástico. Los especímenes con sustituciones del $7.5 \%$ presentaron la mejor resistencia a compresión (18.19 MPa) equivalente al $90.5 \%$ del tradicional (20 MPa). Los resultados encontrados muestran información valiosa para la valorización de desechos plásticos marinos en la elaboración de concretos con resistencias moderadas.

Palabras clave: Residuos plásticos; Concretos modificados; Propiedades mecánicas

Recibido: 23 de Febrero de 2020. Aceptado: 11 de Mayo de 2020.

Received: February 23, 2020. Accepted: May 11, 2020.

\section{MECHANICAL PROPERTIES OF MODIFIED CONCRETE WITH RECYCLED MARIN PLASTIC IN REPLACEMENT OF FINE AGREGATES}

\begin{abstract}
Plastic waste and its low recycling rate contribute to environmental pollution, requiring valorization strategies This work presents the design and preparation of traditional and mix concrete with replacement of $2.5 \%, 5.0 \%$, $7.5 \%$ and $10 \%$ of fine aggregates by recycled plastic of the ocean. With the prepared concrete mixes, cylindrical specimens of $15 \mathrm{~cm} \times 30 \mathrm{~mm}$ in diameterxheight, respectively, were made. Slump, density, compressive strength, and elasticity modulus were evaluated. The results exhibited that an increase in the content of recycled plastic generates a decrease in slump and density, as well as an increase in strength and elastic modulus. Specimens with $7.5 \%$ exhibited the highest strength $(18.9 \mathrm{MPa}$ ) equivalent to $91 \%$ of the traditional one $(20 \mathrm{MPa})$. The results gathered would form a part of useful information for recycling plastic waste in the preparation of concrete that requiring moderate strength.
\end{abstract}

Keywords: Plastic waste; modified concrete; mechanical properties.

Cómo citar este artículo: J. Venitez, Y. Córdoba, K. Mena, O. Arbelaez. "Propiedades mecánicas de concretos modificados con plastico marino reciclado en reemplazo de los agregados finos", Revista Politécnica, vol.16, no.31 pp.77-84, 2020. DOI: 10.33571/rpolitec.v16n31a6 


\section{INTRODUCCIÓN}

La gestión inadecuada de desechos sólidos y en particular de los residuos plásticos, se ha convertido en un problema medioambiental y de salud pública [1]. Miles de desechos plásticos son incinerados, liberando cargas elevadas de contaminantes tóxicos como dióxido de carbono $\left(\mathrm{CO}_{2}\right)$, óxidos de nitrógeno (NOx), y metano $\left(\mathrm{CH}_{4}\right)$,principales contribuyentes del efecto invernadero [2], otros, se acumulan en los rellenos sanitarios, agotando rápidamente la vida útil del relleno y generando problemas por contaminación ambiental y en la salud humana [3], otra parte, se vierte en los ríos y luego es transportada largas distancias hasta los mares, donde se estima que cada año se vierten aproximadamente 8 millones de toneladas de material plástico [4], lo cual, se ha convertido en un problema ambiental grave en el siglo XXII [5]. Estados Unidos, Europa y Asia contribuyen con un $40 \%, 45 \%$ y $15 \%$, respectivamente, a la generación de residuos plásticos marinos y se ha informado que el uso de plásticos se ha multiplicado en un factor de veinte en el último medio siglo [6], por tanto, la valorización de estos residuos se ha convertido en un desafío en la mayoría de los países.

Existen varios tipos de residuos plásticos, siendo el polietileno y el tereftalato de polietileno (conocido comúnmente como PET, de sus iniciales en inglés), los más comunes en las corrientes de desechos, sin embargo, su reutilización está ganando cada vez más terreno en la fabricación de ladrillos ecológicos [7], mezclas de concreto [8], mezclas asfálticas [9] y como aislante térmico [10]. Específicamente, el tereftalato de polietileno reciclado ha sido utilizado en la elaboración de concretos ligeros, con densidades entre $300 \mathrm{Kg} / \mathrm{m}^{3}$ y $1850 \mathrm{Kg} / \mathrm{m}^{3}$ [11] y resistencias a la compresión alrededor de $21 \mathrm{Mpa}$, los cuales, permiten reducir las cargas muertas y las fuerzas sísmicas en las estructuras. La densidad y la resistencia a la compresión de los concretos livianos está altamente influenciada por el contenido (alrededor del 60\%) y la granulometría de los agregados finos (tamaño máximo nominal menor a $10 \mathrm{~mm}$ ) [12], de hecho, cuando se desean obtener resistencias superiores a $21 \mathrm{MPa}$ se requiere el uso de aditivos plastificantes, los cuales, incrementan los costos de elaboración, por encima del 5\% con respecto al concreto tradicional [13].

Záleská y colaboradores [14] reportaron la elaboración de concretos modificados a partir de residuos de plástico con tamaños de partícula menores a $8 \mathrm{~mm}$, los autores reportaron que reemplazos del $10 \%, 20 \%, 30 \%, 40 \%$ y $50 \%$ de los agregados finos presentaron resistencias a la compresión del $82 \%, 59 \%, 26 \%, 9 \%$ y $7 \%$, respectivamente, con respecto al concreto tradicional, comportamiento atribuido a la baja resistencia del material plástico y a la poca interacción entre este material y los componentes de la matriz de concreto, dada la naturaleza hidrofóbica del PET [15]. Asimismo, los autores reportaron, que el aumento en el contenido de reemplazo de plástico es inversamente proporcional a la densidad de los especímenes preparados, de hecho, para reemplazos del $10 \%, 20 \%, 30 \%, 40 \%$ y $50 \%$, se observaron disminución en la densidad del 10\%, $22 \%, 36 \%, 49 \%$ y del $57 \%$, respectivamente, tendencia atribuida a la menor densidad del plástico reciclado en relación a los agregados finos. Asimismo, Alqahtani y colaboradores [15], evaluaron el reemplazo del $25 \%, 50 \%, 75 \%$ y $100 \%$ de los agregados finos por plástico reciclado, encontrando que un reemplazo del $25 \%$, es el más adecuado, presentando una resistencia de $26.9 \mathrm{MPa}$, equivalente al $89 \%$ de la resistencia del concreto tradicional (30 Mpa). Thorneycroft y colaboradores [16], también prepararon concretos con reemplazos del $10 \%$ de los agregados finos por plástico reciclado, los autores reportaron un aumento del $1.2 \%$ de la resistencia a la compresión por encima del material tradicional. Marzouk y colaboradores [17] reportaron la utilización de botellas de tereftalato de polietileno como sustitutos de los agregados finos, con reemplazos entre el $2 \%$ y el $100 \%$ en la preparación de concretos de los agregados finos, los autores, reportaron que un reemplazo del $50 \%$, es el más adecuado, mostrando una disminución de la resistencia a la compresión $16 \%$ en relación al sistema de referencia. Asimismo, los autores reportaron que, en este mismo rango de reemplazo, para sustituciones entre el $2 \%$ y $100 \%$, se observó una disminución de la densidad entre el $5 \%$ y el $50 \%$, respectivamente, de hecho, los autores, concluyen que la densidad es altamente influenciada por el contenido de material reciclado.

Dada la elevada producción de desechos plásticos sin planes adecuados de reutilización y valorización, además del potencial uso de este material en la generación de concretos modificados, con resistencias alrededor de $20 \mathrm{Mpa}$, en este trabajo se presenta el diseño y la preparación de mezclas de concreto tradicional y modificado con reemplazos del $2.5 \%, 5 \%, 7 \%$ y $10 \%$ de los agregados finos por plástico reciclado del océano. A partir de las mezclas 
preparadas, se elaboraron especímenes cilíndricos de $15 \mathrm{~cm} \times 30 \mathrm{~cm}$ de diámetroxlongitud, respectivamente. Se evaluaron las propiedades en estado fresco (asentamiento) y endurecido (densidad, resistencia a compresión y modulo de elasticidad) de los especímenes elaborados. El presente trabajo apunta a potencializar la reutilización de desechos plásticos, en la producción de concretos livianos y con resistencias moderadas a la compresión, en aras de minimizar el efecto negativo de este tipo de residuos sobre el medio ambiente.

\section{MATERIALES Y METODO}

\subsection{Caracterización de los materiales}

Para la preparación de las mezclas se utilizó cemento portland de uso general con una densidad especifica de $3150 \mathrm{Kg} / \mathrm{m}^{3}$ y agua del acueducto. La caracterización de los materiales pétreos se realizó de acuerdo a las especificaciones de la norma técnica colombiana NTC, tal como se describe a continuación: masa unitaria compacta NTC 92 [18], densidad aparente del agregado fino NTC 237 [19], densidad aparente del agregado grueso NTC 176 [20] y granulometría de los agregados NTC 77 [21]. Para el diseño de las mezclas se utilizó una relación agua/cemento de 0.58 . El material sustituto de los agregados finos, provino de la zona costera del municipio de Turbo, donde se hizo la recolección y la clasificación del material, específicamente botellas de plástico. El material recolectado se cortó, y se fundió en un horno eléctrico Herotec CR-35, utilizando una rampa de calentamiento de $5^{\circ} \mathrm{C} / \mathrm{min}$ hasta $180^{\circ} \mathrm{C}$, una vez alcanzada esta temperatura se mantuvo durante $1.5 \mathrm{~h}$. El sólido fundido se presionó en un sistema hidráulico uniaxial a una tonelada métrica durante 15 min y luego se cortó en pellets a un tamaño promedio de $4 \mathrm{~mm}$. El material elaborado presentó una densidad aparente de $67 \mathrm{Kg} / \mathrm{m}^{3}$.

\subsection{Diseño de la mezcla}

La mezcla de concreto tradicional se diseñó para alcanzar una resistencia a la compresión de $21 \mathrm{MPa}$ (a los 28 días), tal como la describe la metodologia ACI 211.18 (American Concrete Institute) [22]. Se diseñaron 5 mezclas; una mezcla de referencia y 4 mezclas con reemplazos del $2.5 \%, 5 \%, 7.5 \%$ y $10 \%$ en masa de los agregados finos, se prepararon tres especímenes de concreto por cada mezcla.

\subsection{Preparación de las mezclas de concreto}

La preparación de las mezclas de concreto se llevó a cabo tal como lo describe Valencia y colaboradores [23], la cual se describe a continuación; el agregado grueso se humedeció hasta la condición SSS Saturado Superficialmente Seco y se pesó la cantidad requerida en una balanza de precisión. Previo a la preparación de las mezclas, la concretadora se humedeció, y en esta, se mezclaron el agregado grueso, el agregado fino y el PET por tres minutos hasta obtener una mezcla homogénea. Después se adicionó el cemento y el $75 \%$ del agua y se mezcló durante 3 minutos. Cuando se visualizaba una pasta cohesiva y fluida se agregaba el plástico reciclado y el $25 \%$ de agua restante y se mezcló por 4 minutos más. Para analizar las propiedades del concreto fresco se realizó la prueba de asentamiento tal como lo describe la norma técnica colombiana NTC 396 [24].

\subsection{Preparación de los especímenes de concreto tradicional y modificado}

Se prepararon 3 especímenes cilíndricos de $15 \mathrm{~cm} \times 30 \mathrm{~cm}$ de diámetroxaltura, respectivamente, como se describe en la norma técnica colombiana NTC 550 [25]. Previo a la preparación de los especímenes, el interior de los moldes se recubrió con una capa fina de desmoldante Separol Ecológico 140902 para prevenir la adherencia del concreto. Los moldes se llenaron en tres capas iguales compactadas con 25 golpes distribuidos en toda la superficie con una varilla de $16 \mathrm{~mm}$ de diámetro. Posteriormente, el molde se golpeó 15 veces con un martillo de goma con el propósito de eliminar las burbujas de aire de cada una de las capas. La última capa se enrasó con la varilla. Para mantener la humedad de los especímenes, la superficie de los moldes se cubrió con láminas de polietileno.

\subsection{Curado de los especímenes cilíndricos}

Pasadas 24 horas se retiraron los moldes y los especímenes de concreto se sumergieron completamente en agua saturada con oxido de calcio $(3 \mathrm{~g} / \mathrm{L})$ a temperatura ambiente durante 28 días, tal como lo describe la norma técnica colombiana NTC 550. 


\subsection{Evaluación de las propiedades físicas y mecánicas de los concretos elaborados}

La resistencia a compresión $f_{c}^{\prime}$ se realizó por triplicado en una prensa hidráulica $\mathrm{HM}$ de compresión de $1500 \mathrm{KN}$ con una velocidad de carga de $0,6 \mathrm{MPa} / \mathrm{s}$. El módulo de elasticidad y la densidad de los especímenes se evaluaron tal como se describe en las ecuaciones (1) y (2) respectivamente.

$$
\begin{array}{lll}
E_{c}=3900 \sqrt{f^{\prime}} & {[\mathrm{MPa}]} & \text { Ec. } 1 \\
\rho=\frac{M}{V} & {\left[\mathrm{Kg} / \mathrm{m}^{3}\right]} & \text { Ec. } 2
\end{array}
$$

Donde $f_{c}^{\prime}$ es la resistencia a la compresión del concreto en $\mathrm{MPa}$, Ec, módulo de elasticidad en $\mathrm{MPa}$, $\rho$ la densidad del concreto en $\mathrm{Kg} / \mathrm{m}^{3}$, M la masa del espécimen de concreto en $\mathrm{Kg}$ y $\mathrm{V}$ volumen del cilindro en $\mathrm{m}^{3}$.

\section{RESULTADOS Y ANALISIS}

Los resultados de la caracterización de los agregados de muestran en la tabla 1.

Tabla 1. Resultados de caracterización de los agregados

\begin{tabular}{ccc}
\hline $\begin{array}{c}\text { Característica } \\
\text { del material }\end{array}$ & $\begin{array}{c}\text { Agregado } \\
\text { Grueso }\end{array}$ & $\begin{array}{c}\text { Agregado } \\
\text { Fino }\end{array}$ \\
\hline Tamaño máximo* $^{*}$ & 25 & n.a. \\
Tamaño máximo nominal $^{*}$ & 25 & n.a. \\
Modulo de finura ${ }^{*}$ & n.a. & 3,17 \\
Masa Unitaria suelta* $^{* *}$ & 1530 & 1550 \\
Masa unitaria compactada** $^{*}$ & 1640 & 1740 \\
Densidad aparente $^{* \star *}$ & 2780 & 2710 \\
Absorción $^{* * *}$ & $1,0 \%$ & $0,9 \%$ \\
Humedad natural $^{* * *}$ & $2,0 \%$ & $2,0 \%$ \\
\hline n.a: no aplica, ${ }^{*}(\mathrm{~mm}),{ }^{* *}\left(\mathrm{Kg} / \mathrm{m}^{3}\right),{ }^{* *}(\%)$ &
\end{tabular}

La tabla 1 muestra que tanto la distribución del tamaño de los agregados gruesos y finos, así como sus masas unitarias se encuentran dentro de los valores recomendados para la elaboración de concretos.

La variación del asentamiento del concreto fresco en función del contenido de tereftalato de polietileno se presenta en la Figura 1.

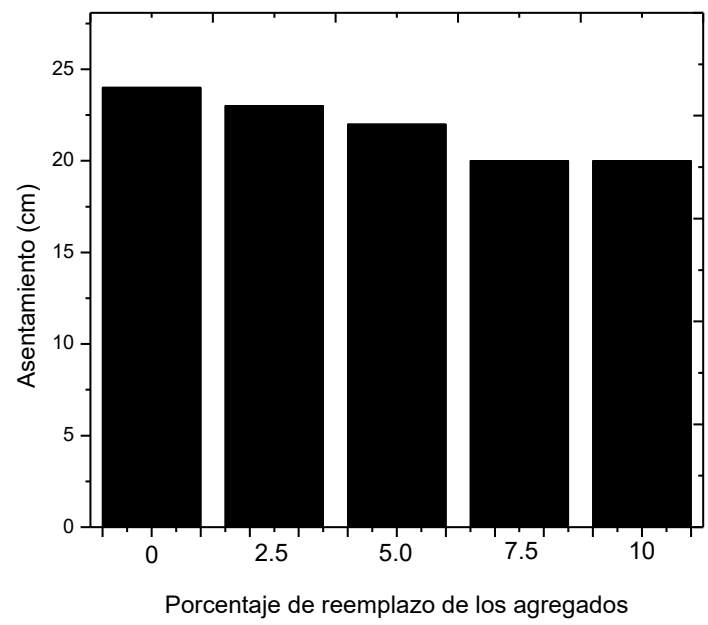

Figura 1. Asentamiento de los especímenes preparados en función del reemplazo de tereftalato de polietileno

Como se muestra en la Figura 1, el asentamiento es influenciado por el contenido de tereftalato de polietileno, disminuyendo con el aumento en el contenido de material reciclado, se observa que un aumento en el reemplazo de PET del $2.5 \%, 5 \%$, $7.5 \%$ y $10 \%$ genera una disminución del $5 \%$, $8 \%$, $16 \%$ y $17 \%$ respectivamente, en relación al material tradicional, el cual presenta un asentamiento de 24 $\mathrm{cm}$, este fenómeno es atribuido a la forma irregular en la cual fue procesado el material reciclado, lo que genera un aumento de la fricción entre las partículas y un disminución en la fluidez de la mezcla. Nuestros resultados están acordes con los reportados por Batayneh y colaboradores [26], quienes reportaron que un aumento en el reemplazo de los agregados finos por partículas de plástico, genera una disminución del asentamiento, de hecho, los autores reportaron que para un reemplazo máximo del $20 \%$ de los agregados finos se observó una disminución del $25 \%$ en el asentamiento, con respecto a la muestra tradicional. Asimismo, nuestros resultados están en línea con los reportados por Ismail y colaboradores [27], quienes reportaron disminuciones del $68 \%, 88 \%$ y $95 \%$ en el asentamiento, para reemplazos del 10\%, 15\% y $20 \%$, respectivamente de los agregados finos por desechos de PET. Los autores atribuyeron la disminución del asentamiento a las formas irregulares de los plásticos reciclados, los cuales presentaron bordes más afilados que el agregado fino, generando una menor fluidez de la mezcla, reflejada en una disminución en el asentamiento. 
Los valores del asentamiento encontrados en este trabajo pueden considerarse aceptables en el rango de reemplazo evaluado. En general, los especímenes preparados con reemplazo de los agregados finos producen una trabajabilidad favorable en términos de facilidad de manejo, colocación y acabado. De hecho, la trabajabilidad se encuentra dentro de los limites (asentamiento $=0$ $25 \mathrm{~mm}$ ) para aplicaciones en concreto vibrado en carreteras y en concretos bombeables.

Los resultados de la densidad de los especímenes preparados en estado endurecido (a los 28 días) se muestran en la Figura 2.

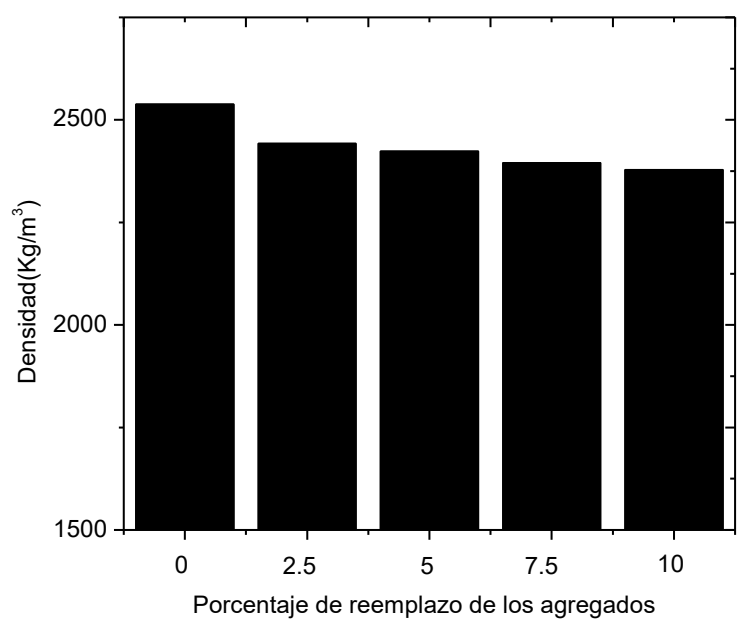

Figura 2. Densidad de los especímenes preparados en función del reemplazo de los agregados finos.

De la figura 2 se puede observar que un aumento en el contenido de PET reciclado, disminuye la densidad de los especímenes preparados, se observa que para porcentajes de reemplazo de PET del $2.5 \%, 5 \%, 7.5 \%$ y $10 \%$ las densidades fueron del $96 \%, 94 \%, 91 \%$ y $90 \%$ de la densidad del concreto tradicional, lo cual se atribuye a la menor densidad del plástico reciclado $\left(67 \mathrm{Kg} / \mathrm{m}^{3}\right)$, la cual representa solo un $2.5 \%$ de la densidad de los agregados finos $\left(2710 \mathrm{Kg} / \mathrm{m}^{3}\right)$. Nuestros resultados son acordes a los reportados por Zainab y colaboradores [27], quienes reportaron una relación inversa entre el contenido de plástico reciclado y la densidad del concreto endurecido, de hecho, los autores encontraron a los 28 días, disminuciones en la densidad de los concretos del $4 \%, 7 \%$ y $9 \%$ para sustituciones del $10 \%, 15 \%$ y $20 \%$, respectivamente de los agregados finos por plástico reciclado. Los autores asociaron esta disminución en la densidad de los concretos con el aumento del contenido de plástico reciclado a la menor densidad, la cual representó solo un $69.7 \%$ de densidad de los agregados finos. De la misma manera, nuestros resultados estuvieron en línea con los reportados por Akçaözog lu y colaboradores [28], quienes encontraron una reducción en la densidad de concretos preparados a partir de residuos de botellas de PET trituradas en reemplazo de los agregados finos, dada la menor gravedad especifica de los residuos de plástico en relación a los agregados. Así mismo, se ha reportado que la sustitución de agregados finos por tereftalato de polietileno (material de naturaleza no polar), genera una tendencia a repeler el agua y atrapar aire dentro de su superficie [29]. Rashid y colaboradores [30] reportaron que un $14 \%$ de aire atrapado reduce la resistencia del concreto en un $45 \%$ por tanto, un aumento en el contenido de tereftalato de polietileno, genera un mayor contenido de aire atrapado en la mezcla, lo cual permite un aumento en la porosidad y una disminución de la densidad tal como se observó en los resultados encontrados.

La resistencia a la compresión de los especímenes preparados se muestra en la Figura 3.

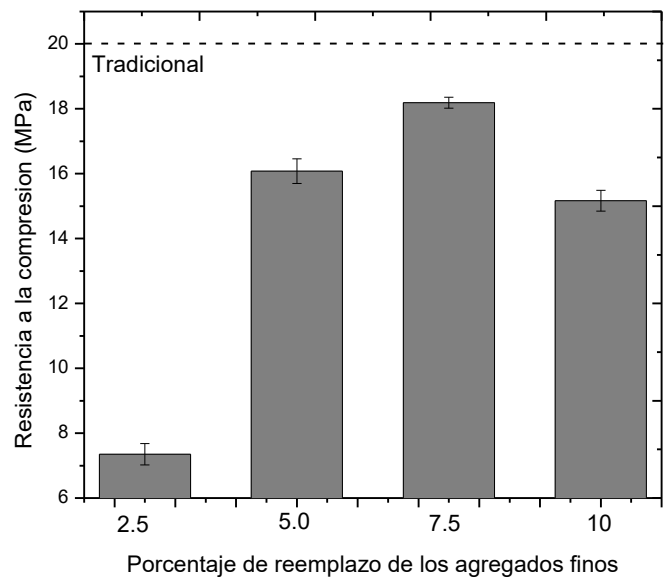

Figura 3. Resistencia a la compresión de los especímenes preparados en función del reemplazo de los agregados finos.

Los resultados de la Figura 3 muestran que la resistencia a la compresión está directamente relacionada con el contenido de material reciclado, se observa que un aumento en el contenido de reemplazo de material reciclado en un rango entre $2.5 \%$ y $7.5 \%$ genera un aumento en la resistencia a la compresión, alcanzando un valor máximo de 18.19 Mpa para un reemplazo de $7.5 \%$, la cual representa el $90.5 \%$ de la resistencia del material 
tradicional, la cual se encuentra en un valor superior a la resistencia a la compresión mínima requerida para un concreto estructural (17.24 Mpa), este comportamiento se atribuye a la fuerza de cohesión entre los residuos plásticos y la pasta de cemento, así como también a la naturaleza hidrofóbica del material reciclado, la cual puede restringir la entrada de agua necesaria para la hidratación del cemento a través de los poros del concreto durante el proceso de curado. De la Figura 3, se puede apreciar que un reemplazo superior al $7.5 \%$ genera una disminución en la resistencia a la compresión. En el rango de reemplazo entre $2.5 \%$ y $7.5 \%$ se encontró una tendencia contraria a la reportada por Batayneh y colaboradores [26] quienes reportaron que en todo el rango de reemplazo, la resistencia a la compresión disminuyó con el aumento en el contenido de PET reciclado. Los resultados reportados por Batayneh y colaboradores [26], reportaron que reemplazos del $5 \%$ y del $10 \%$ de los agregados finos por plástico reciclado generan disminuciones del $23 \%$ y del $38.8 \%$ en la resistencia a la compresión, los cuales fueron mayores a las disminuciones encontradas en este trabajo, donde se observó que para los especímenes del 5\%, 7.5\% y $10 \%$ se observaron disminuciones del $20 \%, 9.5 \%$ y $22.5 \%$ en la resistencia a la compresión, respectivamente. La diferencia con nuestros resultados puede atribuirse a la alta resistencia obtenida por el plástico reciclado durante su proceso de pelletización, generando una menor deformabilidad de la reportada por Batayneh, quienes solo reportaron la trituración de los residuos en la preparación de los concretos modificados.

La Figura 4 muestra los resultados del módulo de elasticidad de los materiales preparados.

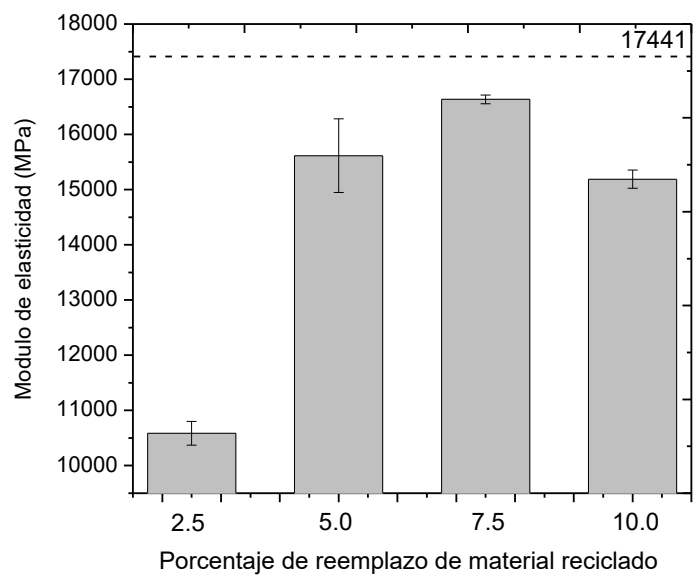

Figura 4. Modulo de elasticidad de los especímenes preparados en función del reemplazo de los agregados finos

El modulo de elasticidad presenta la misma tendencia que la resistencia a la compresión, la cual, acorde a la ecuación 1es directamente proporcional a la compresión. Este comportamiento se atribuye a al aporte individual de cada uno de los materiales constitutivos al módulo de elasticidad del concreto de cada de sus materiales constitutivos. El comportamiento del módulo de elasticidad está relacionado con la reducción de la densidad de los concretos preparados. Este hallazgo indica la influencia positiva de la sustitución de los agregados silíceos con agregados plásticos reciclados.

Con respecto a los costos de producción del concreto, los especímenes cilíndricos con un reemplazo del $7.5 \%$ de los agregados finos por material reciclable, se observó una disminución del $6 \%$ por cada metro cubico de concreto, lo cual, permite determinar que el uso de este tipo de residuos no solo permite la reutilización de materiales de desecho, sino también la reducción de los costos de producción.

\section{CONCLUSIONES}

Se prepararon mezclas de concreto tradicional y modificado con plástico reciclado del océano, en reemplazo de los agregados finos. Se encontró que la modificación de los agregados por plástico reciclado en $2.5 \%, 5.0 \%, 7.5 \%$ y $10 \%$ modifica las propiedades de los concretos preparados. Se encontró que un aumento en el contenido de plástico reciclado, generó una disminución en el asentamiento y en la densidad de los especímenes, como resultado de la forma y la menor densidad de las partículas de PET, respectivamente. Los especímenes de concreto con un reemplazo del $7.5 \%$ de los agregados finos mostraron la mejor resistencia a la compresión, alcanzando un valor de 18.19 Mpa, el cual corresponde al $90.5 \%$ de la resistencia del material tradicional. Los resultados encontrados estuvieron en concordancia con el cambio en el módulo de elasticidad de los especímenes preparados, el cual fue directamente proporcional al aumento en el contenido de plástico reciclado. Los resultados encontrados permiten concluir que los especímenes con reemplazos del $7.5 \%$, permiten generar concretos con resistencias moderadas, entre $18-20 \mathrm{Mpa}$. Se espera que los resultados reportados permitan reducir el uso de 
materiales tradicionales utilizados en la preparación de concretos. Además, se espera que esta metodología sea un nuevo aporte para la generación de concretos de baja densidad y resistencia a la compresión superiores a la resistencia mínima requerida para concretos estructurales, además de mitigar la problemática ambiental generada por la producción de residuos plásticos.

\section{AGRADECIMIENTOS}

Los autores agradecen a la Universidad Cooperativa de Colombia por el apoyo financiero a través del proyecto INV 2731, Venites, J., Córdoba, Y., y Mena, $\mathrm{K}$. agradecen especialmente al semillero de investigación en materiales y estructuras SIMES de la Universidad Cooperativa de Colombia.

\section{REFERENCIAS BIBLIOGRÁFICAS}

[1] Remojan, S. K., Ramya, C., Viswanathan, M. R., Varjani, S. Plastic Pollutants: Waste management for pollution control and abatement, Curren Opin Env Sci., 12, 72-84, 2019.

[2] Da Silva, L. J., Dos Santos, I. F. S., Mensah J. H., Gonçalves, A. T., Barros, R Incineration of municipal solid waste in Brazil: An analysis of the economically viable energy potential, Renew Energ. 149, 1386$1394,2020$.

[3] Awoyera, P.O., Andesina. A. Plastic wastes to construction products: Status, limitations and future perspective, Case Stud. Constr. Mater. 12, e00330 in press, 2020.

[4] Mansour, A. M., Ali, S. A. Reusing waste plastic bottles as an alternative sustainable building material, Energy Sustain Dev 24, 79-85,2015.

[5] Li, J., Lusher, A. L., Rotchell, J. M., Deudero. S., Turra, A., Bråte, I. L. N., Sun, C., Hossain, M. S., Li, Q., Kolandhasamy, P., Shi, H. Using mussel as a global bioindicator of coastal microplastic pollution., 244, 522- 533, 2019.

[6] Geyer, R., Jambeck, J. R., Law, K. L. Production, use, and fate of all plastics ever made, Sci. Adv., 3. 19-24, 2017.

[7] Singh, N., Hui, D., Singh, R., Ahuja, I. P. S., Feo, L., Fraternali, F. Recycling of plastic solid waste: A state of art review and future applications, Compos. B. Eng., 115, 409-422, 2017.
[8] Mohammed, A. A. Modelling the mechanical properties of concrete containing PET waste aggregate, Constr Build Mater., 150, 595-605, 2017.

[9] El-Naga, I. A., Ragab, M. Benefits of utilization the recycle polyethylene terephthalate waste plastic materials as a modifier to asphalt mixtures, Constr Build Mater, 219, 81-90, 2019.

[10] Choudhary, J., Kumar, B., Gupta, A. Utilization of solid waste materials as alternative fillers in asphalt mixes: A review, Constr Build Mater, 234, 117271, 2020.

[11] Tiong. H. Y, Lim. S. K, Lee. Y. L, Ong. C. F, and Yew. M. K, Environmental impact and quality assessment of using eggshell powder incorporated in lightweight foamed concrete, Constr. Build. Mater., 244, 118341, 2020.

[12] Julián. C, Sergio M. A, y William. A, Propiedades mecánicas del concreto para viviendas de bajo costo, Ing. Investig. y Tecnol., 14, 285-298, 2013.

[13] Yoon, J.Y., Kim, J.H. Mechanical properties of preplaced lightweigth aggregates concrete. Constr. Build. Mater, 216, 440-449, 2019.

[14] Záleská. M, Pavlíková. M, Pokorný. J, Jankovský. O, Pavlík. Z, y Černý. R, Structural, mechanical and hygrothermal properties of lightweight concrete based on the application of waste plastics, Constr. Build. Mater., 180, 1-11, 2018.

[15] Alqahtani. F. K, Ghataora. G, Khan. M. I, y Dirar. $S$, Novel lightweight concrete containing manufactured plastic aggregate, Constr. Build. Mater., 148, 386-397, 2017.

[16] Thorneycroft. J, Orr. J, Savoikar. P, and Ball. R. $J$, Performance of structural concrete with recycled plastic waste as a partial replacement for sand, Constr. Build. Mater., 161, 63-69, 2018.

[17] Marzouk. O. Y, Dheilly. R. M, y Queneudec. M, Valorization of post-consumer waste plastic in cementitious concrete composites, Waste Manag., 27, 310-318, 2007.

[18] Instituto Colombiano de Normas Técnicas y Certificación, NTC 92 Determinación de la masa unitaria y los vacíos entre partículas y agregados. 
Colombia,13, 1995.

[19] Instituto Colombiano de Normas Técnicas y Certificación, NTC 237 Método para determinar la densidad y la absorción del agregado fino. Colombia, 14,1995 .

[20] Instituto Colombiano de Normas Técnicas y Certificación, NTC 176 Método de ensayo para determinar la densidad y la absorción del agregado grueso. Colombia, 14, 1995.

[21] Instituto Colombiano de Normas Técnicas y Certificación, NTC 77 Método de ensayo para el análisis por tamizado de los agregados finos y gruesos, no. 571. Colombia, 15, 2007.

[22] ACI Committee, ACI 211.1-91 Standard Practice for Selecting Proportions for Normal, Heavyweight, and Mass Concrete, no. 9. Unites States, 120-121, 2002.

[23] Valencia, J. P. González, A. M, y Arbelaez, O. F, Evaluación de las propiedades mecánicas de concretos modificados con microesferas de vidrio y residuos de llantas, Lámpsakos, 22, 16-26, 2019.

[24] Instituto Colombiano de Normas Técnicas y Certificación, NTC 396 Método de ensayo para determinar el asentamiento del concreto. Colombia, 6, 1992.

[25] Instituto Colombiano de Normas Técnicas y Certificación, NTC 550 Elaboración y curado de especímenes de concreto en obra. Colombia, 13, 2000.

[26] Batayneh. M, Marie. I, y Asi. I, Use of selected waste materials in concrete mixes, Waste Manag., 27, 1870-1876, 2007.
[27] Ismail. Z. Z y AL-Hashmi. E. A, Use of waste plastic in concrete mixture as aggregate replacement, Waste Manag., 28, 2041-2047, 2008.

[28] Akçaözoğlu. S, Atiş.C. D, y Akçaözoğlu. K, An investigation on the use of shredded waste PET bottles as aggregate in lightweight concrete, Waste Manag., 30, 285-290, 2010.

[29] Bing. C y Ning. L, Experimental Research on Properties of Fresh and Hardened Rubberized Concrete, J. Mater. Civ. Eng., 26, 4014-4040, 2013.

[30] Rashid. K, Yazdanbakhsh. A, y UI. M, Sustainable selection of the concrete incorporating recycled tire aggregate to be used as medium to low strength material, J. Clean. Prod., 224, 396-410, 2019. 